International Journal of Pure and Applied Mathematics

Volume 85 No. 6 2013, 1039-1050

ISSN: 1311-8080 (printed version); ISSN: 1314-3395 (on-line version)

url: http://www.ijpam.eu

doi: http://dx.doi.org/10.12732/ijpam.v85i6.7



\title{
MODULATION WAVE EQUATION FOR SPRING-BLOCK ORBITS WITH NONLINEAR RATE AND STATE FRICTION
}

\author{
Kodwo Annan \\ Department of Mathematics \& Computer Science \\ Minot State University \\ Minot, North Dakota, 58707, USA
}

\begin{abstract}
A four-dimensional parameter model that captures the essence of spring-block propagation with rate- and state- friction laws was stated. In order to establish the existence of waves phenomena for all velocity-dependent critical value of stiffness and stick-slip amplitude for stiffness variations, we derived the modulation equation for our model. Conditions under which the amplitude of the modulation wave equation produces many small solutions near the instability threshold for finitely large enough time scales were established.
\end{abstract}

Key Words: spring-block, modulation, rate-state-friction, instability threshold, existence

\section{Introduction}

Non-linear frictional spring-block model systems have been extensively investigated in search of a better understanding and accurate prediction of earthquake dynamics [1-7]. However, a spring-block system cannot replicate an actual earthquake fault without a wealth of laboratory-scale experimental friction data to describing fully these stick-slip processes. Since the introduction of the laboratory determined friction laws (rate- and state- friction law) by Dieterich and Ruina in the late $70 \mathrm{~s}[8,9]$, it's been established that both velocity dependence of friction and time dependent of static friction were important in understanding and predicting earthquake dynamics.

Received: April 13, 2013

(c) 2013 Academic Publications, Ltd. url: www.acadpubl.eu 
The basic idea underlying spring-block models with rate- and state- friction (RSF) laws is that the system considers the effects of inertia and deformations through the introduction of masses and springs. The rate implied instantaneous rate of deformation which is dependent on the friction laws while the state determined the system's internal state which depended on the friction laws. The aforementioned details of the system include such effects as, e.g., dependence of friction on environmental conditions (temperature, humidity, presence of lubricant), history of friction contact (memory effect), wear of materials and surface roughness [10-16]. However, while earthquakes are recurrent and aperiodic on a continuum time scale, the stick-slip of spring-block oscillations has mostly been periodic on a short time scale

Even though few models of RSF capture the essence of stick-slip propagations from difference in values between the static and dynamic coefficients of friction $[6,10,17-21]$, they cannot account for the existence of a velocitydependent critical value of stiffness [17] and the increase of stick-slip amplitude for decreasing stiffness [6, 18, 20-21]. Annan (2012), for example, proved the existence of periodic traveling wave for non coupled, $\ell=0$, and for small perturbation, $\ell \approx 0$ cases [6]. However, to establish the existence of waves phenomena for all $\ell$ we need to examine the modulated waves instead of the basic periodic traveling waves.

In this paper, we derive the modulated wave orbits for spring-block model with non-linear RSF law that allows the amplitude of the wave to vary slowly with time and space. Conditions under which the amplitude of the modulated wave equation produces many small solutions near the instability threshold for finitely large enough time scales are established.

The rest of the paper is arranged as follows: The 4-dimensional parametric model of spring-block propagation with rate and state dependent friction law was stated in Section 2. The derivation of the modulation wave equation is established in Section 3. Conditions under which the modulation equation generated many small solutions near the instability threshold for finitely large enough time scales were established in Section 4. We then give the conclusion in Section 5.

\section{Model Formulation}

We adapt the dimensionless spring-block model derived in [6] for the Burridge and Knopoff model [2] where the friction force is replaced by rate- and state- 
dependent friction laws and $x_{j}(t), \theta_{j}(t) \in \mathbb{R}$ as

$$
\ddot{x}_{j}+x_{j}-\ell^{2}\left(x_{j+1}-2 x_{j}+x_{j-1}\right)=F\left(\dot{x}_{j}, \theta_{j}\right) ; j \in \mathbb{Z} .
$$

Since the state variable, $\theta_{j}$, is time-dependent, we must have a time evolution law for $\theta_{j}$ together with the friction force. Here, we assume the dimensionless form of the Dieterich's law (also known as slowness law) [9] to describe the time-dependent properties of the friction law as

$$
\dot{\theta}_{j}=1-\theta_{j}\left(V+\dot{x}_{j}\right)
$$

Thus, (2) describes the time-dependent increase of the state variable even at $V=0$ where $V$ is the dimensionless loading rate. Following the model derivation of spring-block model with RSF law presented in $[3,22]$, the system equation is presented as

$$
\left\{\begin{array}{c}
\ddot{X}_{j}+X_{j}=\ell^{2}\left(X_{j+1}-2 X_{j}+X_{j-1}\right)+a_{1} \dot{X}_{j}+b_{1} \psi_{j}+\Gamma\left(\dot{X}_{j}, \psi_{j}\right) \\
\dot{\psi}_{j}=\alpha_{1} \dot{X}_{j}+\alpha_{2} \psi_{j}+\alpha_{3} \psi_{j} \dot{X}_{j}, \text { for } j \in \mathbb{Z}
\end{array}\right.
$$

where $\Gamma\left(\dot{X}_{j}, \psi_{j}\right)=a_{2} X^{2}+a_{3} X^{3}+o\left(X^{4}\right)+b_{2} \psi^{2}+b_{3} \psi^{3}+o\left(\psi^{4}\right), a_{1}=-a(1+V)^{-1}$, $b_{1}=-b V$ and $a_{i}, b_{i}, \alpha_{i}$ for $i=1,2,3$ are dimensionless parameters. Since $a_{1}, b_{1}, \alpha_{1}$ and $\alpha_{2}$ are negative, we make a hypothesis that

$$
a_{1}, b_{1}, \alpha_{1} \text { and } \alpha_{2} \text { are non - positive. }
$$

Thus, the system (3) depends on a four-dimension parameter $\mathrm{P}=\left(a_{1}, b_{1}, \alpha_{1}, \alpha_{2}\right)$.

In his paper, Annan (2013) developed the general setup of equation (3) using spectral method approach to describe the model in space and time of a spring-block periodic pattern waves close to the critical variety of parameter space for small $\ell$. The method will not work well in a case where we are interested in a large class of solutions for all $\ell$. Therefore, instead of using a periodic propagation of waves approach, we develop a modulated wave equation for our model. The general idea of modulation is that if we consider the physical domain $\xi=\mathbb{R}^{d} \times \Sigma$, where $\Sigma$ is a bounded cross-section, with $\varepsilon \in \mathbb{R}^{d}$ and $r \in \Sigma$, then the basic pattern of (3) takes the form

$$
p(t, \varepsilon, r)=\varphi_{0, k_{0}}(r) \lambda(t, \varepsilon) \text { where } \lambda(t, \varepsilon)=e^{i\left(\omega t+\left\langle k_{0}, \varepsilon\right\rangle\right)} .
$$

Here, $\omega$ and $k_{0}$ belonging to $\mathbb{R}^{d}$ denote the temporal frequency and the spatial wave vector of the basic pattern, respectively. Therefore, the modulation pattern equation is of the form

$$
\hbar^{A}(t)=A(\tau, \varsigma) p(t, x)+C_{c},
$$


where the amplitude function $A(\tau, \varsigma) \in \mathbb{C}$ depends on a slow time variable $\tau$ and a large spatial variable $\varsigma$. The amplitude $|A(\tau, \varsigma)|$ gives the intensity of the basic pattern while phase changes of $A$ give rise to changes of the pattern in the temporal frequency and in the spatial wave vector.

\section{Modulation Equation Derivation}

Let $\lambda(\varepsilon, r)$ and $\varphi_{\varepsilon, r}$ be the eigenvalue and eigenvector of the linear operator $\mathcal{L}(\varepsilon, i r)$ derived in $[3]$, then $X=\varphi_{\varepsilon, r}\left[e^{\lambda(\varepsilon, r) t+i r j}\right]_{j}$ is a solution to the linear equation. If the solution has a single pair of eigenvalues that crosses the complex plane at $\varepsilon=0$, then for a certain $r_{0}$ we have $\lambda\left(0, r_{0}\right)=i \omega$. Thus, we can write the expression for the largest real eigenvalue for $\mathcal{L}(\varepsilon, i r)$ as [23] by

$$
\lambda(\varepsilon, r)=\lambda_{0}(\varepsilon)\left(r-r_{0}\right)+\lambda_{1}(\varepsilon)\left(r-r_{0}\right)^{2}+\lambda_{2}(\varepsilon)\left(r-r_{0}\right)^{2}+o\left(\left|r-r_{0}\right|^{3}\right) .
$$

If we cross the imaginary axis from left to right at $\varepsilon=0$ and consider $\operatorname{Re}\left(\lambda_{0}(0)\right)=$ $0, \frac{d \operatorname{Re}\left(\lambda_{0}(0)\right)}{d \varepsilon}>0$ and $\operatorname{Re}\left(\lambda_{1}(0)\right)=0$, then for $(\varepsilon, r) \rightarrow\left(0, r_{0}\right)$ we have

$$
\varepsilon=\rho \delta^{2} \text { for } \rho= \pm 1 \text { and } r=r_{0}+\delta \kappa \text {. }
$$

Thus, (5) reduces to

$$
\lambda(\varepsilon, r)=i \omega+\delta^{2} \rho \lambda_{0,1}+\delta \kappa \lambda_{1}(0)-\delta^{2} \Lambda^{2}(0)+\mathrm{O}\left(\delta^{3}\right),
$$

with the solution of the linear problem in the form

$$
e^{\lambda(\varepsilon, r)+i r j}=e^{\left(\rho \lambda_{0,1}-\Lambda_{0} \kappa^{2}\right) \delta^{2} t+i \kappa \delta\left(j+i \lambda_{1}(0)\right)+\mathrm{O}\left(\delta^{2}\right)} p(t, j) .
$$

Here, we have denoted $p(t, j)$ by $e^{i\left(\omega t+r_{0} j\right) \varphi_{\delta}{ }^{2}, r_{0}+\delta \kappa} \approx e^{i\left(\omega t+r_{0} j\right) \varphi_{0, r_{0}}}$ as the only mode that is not dumped at $\varepsilon=0$. We see that the amplitude of (7) depends on the slow time variable $\delta^{2} t$ and on the slow space variable $\varsigma=\delta\left(j-C_{g} t\right)$ where $C_{g}=-i \lambda_{1}(0)$. So, we expect that there exist solutions to the original non-linear system that are close to $\hbar^{A}(t)$ for small $\varepsilon>0$ if the solution is of the form $\hbar^{A}(t)=A(\tau, \varsigma) p(t, x)+C_{c}$, where $C_{c}$ is the complex conjugates.

In addition, as $\delta^{2} \rightarrow 0$, we expect the modulated waves to be of small amplitude. Therefore, we make a scaling and assume that the amplitude is of order $\mathrm{O}(\delta)$. Hence the modulated wave orbits form of $(3)$ is

$$
\hbar^{A}(t)=\left(\begin{array}{c}
X^{A}(t) \\
\dot{X}^{A}(t) \\
\psi^{B}(t)
\end{array}\right) \text { for }\left\{\begin{array}{l}
X_{j}^{A}(t)=\delta A\left(\delta^{2} t, \delta\left(j-C_{g} t\right)\right) e^{i \omega t+i r_{0} j}+C_{c} \\
\psi_{j}^{B}(t)=\delta B\left(\delta^{2} t, \delta\left(j-C_{g} t\right)\right) e^{i \omega t+i r_{0} j}+C_{c}
\end{array}\right.
$$


We now claim that if the solution close to (8) exists, then substituting the solution into the system (3) and determining the power of $\delta$ will lead to the amplitude equation

$$
\partial_{\tau} A=(c+i d) A+(\vartheta+i \alpha) \partial_{\varsigma \varsigma} A+(a+i b)|A|^{2} A, \quad A(\tau, \varsigma) \in \mathbb{C} .
$$

Proposition 3.1. The group velocity of the modulated wave (8) is zero when $C_{g}=0$.

Proof. By definition $C_{g}=-i \frac{\partial \lambda(0,0)}{\partial r}$. Obtaining the derivative again with the implicit equation

$$
p(\lambda(\varepsilon, r), \varepsilon, r)=0 \Rightarrow \frac{\partial \lambda(0,0)}{\partial r}=\frac{\partial_{r} p(i \omega, 0,0)}{\partial_{X} p(i \omega, 0,0)} .
$$

Since $i \omega$ is a simple root for $\varepsilon=0$ and $r=0$,it implies $\partial_{X} p(i \omega, 0,0) \neq 0$ and so $\partial_{r} p(i \omega, 0,0)=0$.

Also, since we are looking for a solution of the form

$$
\left\{\begin{array}{l}
X_{j}(t)=\delta A\left(\delta^{2} t, \delta j\right) e^{i \omega t}+C_{c}+\mathrm{O}\left(\delta^{2}\right), \\
\psi_{j}(t)=\delta B\left(\delta^{2} t, \delta j\right) e^{i \omega t}+C_{c}+\mathrm{O}\left(\delta^{2}\right) .
\end{array}\right.
$$

We notice from (3) that the non-linear terms contain $e^{2 i \omega t}, e^{3 i \omega t}, \ldots$ terms with amplitude $\delta^{2}, \delta^{3}, \ldots$. Therefore, we introduce the following series approach to account for the terms

$$
X_{j}(t)=\sum_{k=0}^{\infty} \sum_{n \in \mathbb{Z}} \delta^{k} A_{k n}(\tau, \varsigma) e^{i \omega t} ; \quad \psi_{j}(t)=\sum_{k=0}^{\infty} \sum_{n \in \mathbb{Z}} \delta^{k} B_{k n}(\tau, \varsigma) e^{i \omega t},
$$

where the amplitudes $A_{k n}$ and $B_{k n}$ are complex terms $A_{k-n}=\bar{A}_{k n}$ and $B_{k-n}=$ $\bar{B}_{k n}$ for all $k \in \mathbb{N}$ and $n \in \mathbb{Z}$. Also,

$$
\tau=\delta^{2} t \text { (slow time variable) and } \varsigma=\delta j \text { (slow space variable). }
$$

Annan (2013) has shown the existence of a bifurcation in model system (3) at some critical value of the hypothesis parameters (4) at $\Delta^{c}=\left[a_{1}^{c}, b_{1}^{c}, \alpha_{1}^{c}, \alpha_{2}^{c}\right]$. The researcher proved that at $\varepsilon=0$, the spectrum of the linear operator $\mathcal{L}$ has a complex pair of element crossing the imaginary axis at $\pm i \omega$ with $\omega=$ $\sqrt{a_{1}^{c} \alpha_{2}^{c}-\alpha_{1}^{c} b_{1}^{c}+1}$. Specifically, the model system was uniformly stable at the origin but when crossing the threshold of instability at $\Delta^{c}$, there existed a wide range of unstable modes, $r$ [3]. Therefore, the origin is unstable for $\varepsilon>0$. 
Since the origin is unstable for any $\varepsilon>0$, we consider $\varepsilon=+\delta^{2}$ and substitute (10) into model system (3) to have

$$
\left\{\begin{array}{c}
\ddot{X}_{j}+X_{j}=\left(\Delta_{X}\right)_{j}+\left(a_{1}^{c}+a_{11} \delta^{2}+\mathrm{O}\left(\delta^{2}\right)\right) \dot{X}_{j}+a_{2} \dot{X}_{j}^{2}+a_{3} \dot{X}_{j}^{3} \\
+\left(b_{1}^{c}+b_{11} \delta^{2}+\mathrm{O}\left(\delta^{2}\right)\right) \psi_{j}+b_{2} \psi_{j}^{2}+b_{3} \psi_{j}^{3}+N_{1}\left(\dot{X}_{j}\right)+N_{2}\left(\psi_{i}\right) \\
\dot{\psi}_{j}=\left(\alpha_{1}^{c}+\alpha_{11} \delta^{2}+\mathrm{O}\left(\delta^{2}\right)\right) \dot{X}_{j}+\left(\alpha_{2}^{c}+\alpha_{11} \delta^{2}+\mathrm{O}\left(\delta^{2}\right)\right) \psi_{j}+\alpha_{3} \psi_{j} \dot{X}_{j}
\end{array}\right.
$$

where $N_{1}(x), N_{2}(x)=\mathrm{O}\left(x^{4}\right), \Delta_{X}=X_{j+1}-2 X_{j}+X_{j-1}$ and $j \in \mathbb{Z}$.

Theorem 3.2. Suppose that (3) has solutions of the form

$$
\left\{\begin{array} { l } 
{ X _ { j } ( t ) = X _ { j } ^ { A } ( t ) + \mathrm { O } ( \delta ^ { 2 } ) , } \\
{ \psi _ { j } ( t ) = \psi _ { j } ^ { B } + \mathrm { O } ( \delta ^ { 2 } ) , }
\end{array} \quad \text { with } \quad \left\{\begin{array}{l}
X_{j}^{A}(t)=\delta A(\tau, \varsigma) e^{i \omega t}+C_{c} \\
\psi_{j}^{B}=\delta B(\tau, \varsigma) e^{i \omega t}+C_{c}
\end{array}\right.\right.
$$

for all $j \in \mathbb{Z}$ and $\delta \in\left(0, \delta_{0}\right)$, where $A$ is a smooth function $[0, T] \times \mathbb{R} \rightarrow \mathbb{C}$. Then $A$ is a solution to the complex scalar Ginzburg-Landau equation

$$
\partial_{\tau} A=(c+i d) A+(\vartheta+i \alpha) \partial_{\varsigma \varsigma} A+(a+i b)|A|^{2} A, \quad A(\tau, \varsigma) \in \mathbb{C} .
$$

with the following coefficients

$$
\begin{aligned}
& c+i d=\frac{\left(i \omega-\alpha_{2}^{c}\right) b_{11} \Omega_{11}+b_{1}^{c}\left(\beta_{11} \Omega_{11}+i \omega \alpha_{11}\right)}{\left(2 i \omega-a_{1}^{c}\right)\left(i \omega-\alpha_{2}^{c}\right)-b_{1}^{c}\left(\alpha_{1}^{c}-\Omega_{11}\right)} \\
& \vartheta+i \alpha= \frac{\left(i \omega-\alpha_{2}^{c}\right) \ell^{2}}{\left(2 i \omega-a_{1}^{c}\right)\left(i \omega-\alpha_{2}^{c}\right)-b_{1}^{c}\left(\alpha_{1}^{c}-\Omega_{11}\right)} \\
& a+i b=\frac{\left(i \omega-\alpha_{2}^{c}\right)\left(4 a_{2} \omega^{2} \Omega_{22}+2 b_{2} \Omega_{22}^{\prime} \bar{\Omega}_{11}+3 b_{3} \Omega_{11}\left|\Omega_{11}\right|^{2}+3 i \omega^{3} a_{3}\right)}{\left(a_{1}^{c}-2 i \omega\right)\left(i \omega-\alpha_{2}^{c}\right)+b_{1}^{c}\left(\alpha_{1}^{c}-\Omega_{11}\right)} \\
&+\frac{i \omega b_{1}^{c} \alpha_{3}\left(2 \Omega_{22} \Omega_{11}+\Omega_{20}-\Omega_{22}^{\prime}\right)}{\left.\left(a_{1}^{c}-2 i \omega\right)\left(i \omega-\alpha_{2}^{c}\right)+b_{1}^{c}\left(\alpha_{1}^{c}-\Omega_{11}\right)\right)} .
\end{aligned}
$$

and $B$ given by the expression $B=\frac{i \omega \alpha_{1}^{c}}{i \omega-\alpha_{2}^{c}} A$.

Proof. To prove that given a solution to the Ginzburg-Landau equation, we can determine all the amplitudes $A_{k n}$ for $1 \leq k \leq 3$ and $-k \leq n \leq k$, let's consider $X$ and $\psi$ defined in (10) and express the series at order $\delta^{3}$ where $\lambda(t)=e^{i \omega t}$. Then

$$
\begin{aligned}
& \dot{X}_{j}(t)=i \omega A_{11} \delta \lambda+i \omega A_{21} \delta^{2} \lambda+2 i \omega A_{22} \delta^{2} \lambda^{2}+\partial_{\tau} A_{10} \delta^{3} \lambda^{0}+2 i \omega A_{32} \delta^{3} \lambda^{2} \\
& +\left(\partial_{\tau} A_{11}+3 i \omega A_{31}\right) \delta^{3} \lambda+3 i \omega A_{33} \delta^{3} \lambda^{3}+C_{c}+\mathrm{O}\left(\delta^{3}\right), \\
& \dot{X}_{j}^{2}(t)=2 \omega^{2}\left|A_{11}\right|^{2} \delta^{2} \lambda^{0}-\omega^{2} A_{11}^{2} \delta^{2} \lambda^{2}+2 \omega^{2}\left(\bar{A}_{11} A_{21}+A_{11} \bar{A}_{21}\right) \delta^{3} \lambda^{0} \\
& \quad+4 \omega^{2} \bar{A}_{11} A_{22} \delta^{3} \lambda-2 \omega^{2} A_{11} A_{21} \delta^{3} \lambda^{2}-4 \omega^{2} A_{11} A_{22} \delta^{3} \lambda^{3}+C_{c}+\mathrm{O}\left(\delta^{3}\right), \\
& \dot{X}_{j}^{3}(t)=3 i \omega^{2}\left|A_{11}\right|^{2} A_{11} \delta^{3} \lambda-i \omega^{3} A_{11}^{3} \delta^{3} \lambda^{3}+C_{c}+\mathrm{O}\left(\delta^{3}\right)
\end{aligned}
$$




$$
\begin{gathered}
\ddot{X}_{j}(t)=-\omega^{2} A_{11} \delta \lambda-\omega^{2} A_{21} \delta^{2} \lambda-4 \omega^{2} A_{22} \delta^{2} \lambda^{2}-4 \omega^{2} A_{32} \delta^{3} \lambda^{2} \\
+\left(2 i \omega \partial_{\tau} A_{11}-\omega^{2} A_{32}\right) \delta^{3} \lambda-9 \omega^{2} A_{33} \delta^{3} \lambda^{3}+C_{c}+\mathrm{O}\left(\delta^{3}\right), \\
\Delta_{X}(t)=\partial_{\varsigma \varsigma} A_{10} \delta^{3} \lambda^{0}+\partial_{\varsigma \varsigma} A_{11} \delta^{3} \lambda+C_{c}+\mathrm{O}\left(\delta^{3}\right), \\
\psi_{j}^{2}(t)=\left(B_{10}^{2}+2\left|B_{11}\right|^{2}\right) \delta^{2} \lambda^{0}+2 B_{10} B_{11} \delta^{2} \lambda+B_{11}^{2} \delta^{2} \lambda^{2}+2 B_{11} \delta^{3} \lambda^{3} \\
+2\left(B_{10} B_{20}+B_{11} \bar{B}_{21}+\bar{B}_{11} B_{21}\right) \delta^{3} \lambda^{0}+2\left(B_{10} B_{21}+B_{10} \bar{B}_{20}+\bar{B}_{11} B_{22}\right) \delta^{3} \lambda \\
+2\left(B_{10} B_{22}+B_{11} B_{21}\right) \delta^{3} \lambda^{2}+C_{c}+\mathrm{O}\left(\delta^{3}\right), \\
\psi_{j}^{3}(t)=B_{10}^{3} \delta^{3} \lambda^{0}+3\left(B_{10}^{2} B_{11}+B_{11}^{2} \bar{B}_{11}\right) \delta^{3} \lambda+3 B_{11}^{2} B_{10} \delta^{3} \lambda^{2} \\
+B_{11}^{3} \delta^{3} \lambda^{3}+C_{c}+\mathrm{O}\left(\delta^{3}\right), \\
\psi_{j}(t) \dot{X}_{j}(t)=i \omega\left(A_{11} \bar{B}_{11}-B_{11} \bar{A}_{11}\right) \delta^{2} \lambda^{0}+i \omega B_{10} A_{11} \delta^{2} \lambda+i \omega B_{11} A_{11} \delta^{2} \lambda^{2} \\
+i \omega\left(\bar{B}_{11} A_{21}-B_{11} \bar{A}_{21}+A_{11} \bar{B}_{21}-\bar{A}_{11} B_{21}\right) \delta^{3} \lambda^{0} \\
+i \omega\left(B_{10} A_{21}+2 A_{22} \bar{B}_{11}+A_{11} B_{20}-\bar{A}_{11} B_{22}\right) \delta^{3} \lambda^{1} \\
+i \omega\left(2 B_{10} A_{22}+B_{11} A_{21}+A_{11} B_{21}\right) \delta^{3} \lambda^{2} \\
+i \omega\left(2 A_{22} B_{11}+A_{11} B_{22}\right) \delta^{3} \lambda^{3}+C_{c}+\mathrm{O}\left(\delta^{3}\right) .
\end{gathered}
$$

Substituting the above expressions into (3) and comparing each order $\delta^{k} \lambda^{n}$ for $1 \leq k \leq 3$ and $-k \leq n \leq k$ gives the following results

The $\delta \lambda^{0}$ term gives : $\left\{\begin{array}{l}A_{10}=b_{1}^{c} B_{10} \\ 0=\alpha_{2}^{c} B_{10}\end{array} \quad \Rightarrow A_{10}=B_{10}=0\right.$.

The $\delta \lambda$ term gives : $\left\{\begin{array}{l}-\omega A_{11}+A_{11}=a_{1}^{c} i \omega A_{11}+b_{1}^{c} B_{11} \\ i \omega B_{11}=\alpha_{1}^{c} i \omega A_{11}+\alpha_{2}^{c} B_{11}\end{array}\right.$,

$$
\Rightarrow \quad B_{11}=\Omega_{11} A_{11} \quad \text { where } \quad \Omega_{11}=\frac{i \omega \alpha_{1}^{c}}{i \omega-\alpha_{2}^{c}} \in \mathbb{C} .
$$

The first equation is written as $p(i \omega, 0,0) A_{11}=0$, which is satisfied for any $A_{11}$ since $p(i \omega, 0,0)=0$.

$$
\begin{gathered}
\text { The } \delta^{2} \lambda^{0} \text { term gives : }\left\{\begin{array}{c}
A_{20}=b_{1}^{c} B_{20}+A 2 a_{2} \omega^{2}\left|A_{11}\right|^{2}+b_{2}\left|B_{11}\right|^{2} \\
0=\alpha_{2}^{c} B_{20}+\alpha_{3}^{c} i \omega\left(A_{11} \bar{B}_{11}-B_{11} \bar{A}_{11}\right.
\end{array},\right. \\
\Rightarrow\left\{\begin{array}{c}
B_{20}=\Omega_{20}\left|A_{11}\right|^{2}, \quad \Omega_{20}=\frac{2 \omega \alpha_{3}}{\alpha_{3}^{c}} \operatorname{Im} \Omega_{11} \in \mathbb{R}, \\
A_{20}=\Omega_{20}^{\prime}\left|A_{11}\right|^{2}, \quad \Omega_{20}^{\prime}=b_{1}^{c} \Omega_{20}+2 a_{2} \omega^{2}+2 b_{2}\left|\Omega_{11}\right|^{2} \in \mathbb{R} .
\end{array}\right.
\end{gathered}
$$

The $\delta^{2} \lambda^{1}$ term gives : $\left\{\begin{array}{c}-\omega^{2} A_{21}+A_{21}=i \omega a_{1}^{c} A_{21}+b_{1}^{c} B_{21} \\ i \omega B_{21}=i \omega \alpha_{1}^{c} A_{21}+\alpha_{2}^{c} B_{21}\end{array}\right.$, 
From the second equation we gets $B_{21}=\Omega_{11} A_{21}$ and it follows that the first equation can be written as $p(i \omega, 0,0) A_{21}=0$ which is satisfied for any $A_{21}$. The $\delta^{2} \lambda^{2}$ term gives:

$$
\begin{aligned}
& \left\{\begin{array}{c}
-4 \omega^{2} A_{22}+A_{22}=2 i \omega a_{1}^{c} A_{22}+b_{1}^{c} B_{22}-\omega^{2} a_{2} A_{11}^{2}+b_{2} B_{11}^{2} \\
2 i \omega B_{22}=2 i \omega \alpha_{1}^{c} A_{22}+\alpha_{2}^{c} B_{22}+i \omega a_{3} B_{11} A_{11} .
\end{array}\right. \\
& \Rightarrow\left\{\begin{array}{ll}
A_{22}=\Omega_{22}^{\prime} A_{11}^{2}, & \Omega_{22}^{\prime}=\frac{\eta(i \omega)}{p(2 i \omega, 0,0)} \in \mathbb{C}, \\
B_{22}=\Omega_{22} A_{11}^{2}, & \Omega_{22}=\frac{2 i \omega \Omega_{22}^{\prime} \alpha_{1}^{+}+i \omega \alpha_{3} \Omega_{11}}{2 i \omega-\alpha_{2}^{c}} \in \mathbb{C}
\end{array},\right.
\end{aligned}
$$

where $\eta(i \omega)=-2 i \omega^{3} a_{2}+a_{2} \alpha_{2}^{c}+i \omega \Omega_{11}\left(\alpha_{3} b_{1}^{c}+2 b_{2} \Omega_{11}\right)-\alpha_{2}^{c} b_{2} \Omega_{11}^{2}$. To obtain $\delta^{3} \lambda^{0}$, we have

$$
\begin{gathered}
\left\{\begin{array}{c}
A_{30}=b_{1}^{c} B_{30}+2 a_{2} \omega^{2}\left(\bar{A}_{11} A_{21}+A_{11} \bar{A}_{21}\right)+2 b_{2}\left(B_{11} \bar{B}_{21}+\bar{B}_{11} B_{21}\right), \\
0=\alpha_{2}^{c} B_{30}+i \omega \alpha_{3}\left(\bar{B}_{11} A_{21}-B_{11} \bar{A}_{21}+A_{11} \bar{B}_{21}-\bar{A}_{11} B_{21}\right) .
\end{array}\right. \\
\Rightarrow\left\{\begin{array}{c}
B_{30}=4 \Omega_{30} \operatorname{Re}\left(A_{11} \bar{A}_{21}\right), \quad \Omega_{30}=\frac{\omega \alpha_{3}}{\alpha_{2}^{c}} \operatorname{Im} \bar{\Omega}_{11} \in \mathbb{R}, \\
A_{30}=\Omega_{30}^{\prime} \operatorname{Re}\left(A_{11} \bar{A}_{21}\right), \quad \Omega_{30}^{\prime}=\Omega_{30}+a_{2} \omega^{2}+b_{2}\left|\Omega_{11}\right|^{2} \in \mathbb{R} .
\end{array}\right.
\end{gathered}
$$

The $\delta^{3} \lambda^{1}$ term gives:

$$
\left\{\begin{array}{c}
-\omega^{2} A_{31}+2 i \omega \partial_{\tau} A_{11}+A_{31}=\ell^{2} \partial_{\varsigma \varsigma} A_{11}+a_{1}^{c}\left(A_{22} \partial_{\tau} A_{11}+i \omega A_{31}\right)+b_{1}^{c} B_{31} \\
4 a_{2} \omega^{2} \bar{A}_{11} A_{22}+2 b_{2} \bar{B}_{11} B_{22}+3 b_{3} B_{11}^{2} \bar{B}_{11}+3 i \omega^{3} a_{3} A_{11}\left|A_{11}\right|^{2} \\
+b_{11} \Omega_{11} A_{11}+i \omega a_{11} A_{11} \\
i \omega B_{31}+\partial_{\tau} B_{11}=a_{1}^{c}\left(\partial_{\tau} A_{11}+i \omega A_{31}\right)+\alpha_{2}^{c} B_{31}+\beta_{11} B_{11} \\
+i \omega \alpha_{3}\left(2 A_{22} \bar{B}_{11}+A_{11} B_{20}-\bar{A}_{11} B_{22}\right)+i \omega \alpha_{11} A_{11}
\end{array}\right.
$$

Using (15) and (17) and the fact that $p(i \omega, 0,0)=0$, the second equation is reduced to

$$
B_{31}=\lambda_{1} A_{31}+\lambda_{2} \partial_{\tau} A_{11}+\lambda_{3} A_{11}\left|A_{11}\right|^{2}+\lambda_{4} A_{11},
$$

where

$$
\begin{array}{ll}
\lambda_{1}=\frac{i \omega \alpha_{1}^{c}}{i \omega-\alpha_{2}^{c}}, & \lambda_{2}=\frac{\alpha_{1}^{c}-\Omega_{11}}{i \omega-\alpha_{2}^{c}}, \\
\lambda_{3}=\frac{i \omega \alpha_{3}\left(2 \Omega_{22} \bar{\Omega}_{11}+\Omega_{20}-\Omega_{22}^{\prime}\right.}{i \omega-\alpha_{2}^{c}}, & \lambda_{4}=\frac{\beta_{11} \Omega_{11}+i \omega \alpha_{11}}{i \omega-\alpha_{2}^{c}} .
\end{array}
$$

Substituting (19) into the first part of the equation gives

$$
\partial_{\tau} A_{11}=(c+i d) A_{11}+(\vartheta+i \alpha) \partial_{\varsigma \varsigma} A_{11}+(a+i b)\left|A_{11}\right|^{2} A_{11},
$$

With

$$
\begin{aligned}
& c+i d=\frac{\left(i \omega-\alpha_{2}^{c}\right) b_{11} \Omega_{11}+b_{1}^{c}\left(\beta_{11} \Omega_{11}+i \omega \alpha_{11}\right)}{\left(2 i \omega-a_{1}^{c}\right)\left(i \omega-\alpha_{2}^{c}\right)-b_{1}^{c}\left(\alpha_{1}^{c}-\Omega_{11}\right)}, \\
& \vartheta+i \alpha=\frac{\left(i \omega-\alpha_{2}^{c}\right) \ell^{2}}{\left(2 i \omega-a_{1}^{c}\right)\left(i \omega-\alpha_{2}^{c}\right)-b_{1}^{c}\left(\alpha_{1}^{c}-\Omega_{11}\right)},
\end{aligned}
$$




$$
\begin{gathered}
a+i b=\frac{\left(i \omega-\alpha_{2}^{c}\right)\left(4 a_{2} \omega^{2} \Omega_{22}+2 b_{2} \Omega_{22}^{\prime} \bar{\Omega}_{11}+3 b_{3} \Omega_{11}\left|\Omega_{11}\right|^{2}+3 i \omega^{3} a_{3}\right)}{\left(a_{1}^{c}-2 i \omega\right)\left(i \omega \omega-\alpha_{2}^{c}\right)+b_{1}^{c}\left(\alpha_{1}^{c}-\Omega_{11}\right)} \\
+\frac{i \omega b_{1}^{\alpha}\left(2 \Omega_{22} \Omega_{11}+\Omega_{20}-\Omega_{22}^{\prime}\right)}{\left.\left(a_{1}^{c}-2 i \omega\right)\left(i \omega-\alpha_{2}^{c}\right)+b_{1}^{c}\left(\alpha_{1}^{c}-\Omega_{11}\right)\right)} .
\end{gathered}
$$

Finally, $\delta^{3} \lambda^{2}$ and $\delta^{3} \lambda^{3}$ are obtained respectively as

$$
\begin{gathered}
\left\{\begin{array}{c}
-4 \omega^{2} A_{32}+A_{32}=2 i \omega a_{1}^{c} A_{32}+b_{1}^{c} B_{32}-2 \omega^{2} a_{2} A_{11} A_{21}+2 b_{2} B_{11} B_{21}, \\
2 i \omega B_{32}=2 i \omega \alpha_{1}^{c} A_{32}+\alpha_{2}^{c} B_{32}+i \omega a_{3}\left(B_{11} A_{21}+A_{11} B_{21}\right) .
\end{array}\right. \\
\Rightarrow\left\{\begin{array}{c}
A_{32}=\Omega_{32}^{\prime} A_{11} A_{21}, \Omega_{32}^{\prime}=\frac{2\left(b_{2} \Omega_{11}^{2}-a_{2} \omega^{2}\right)\left(2 i \omega-\alpha_{2}^{c}\right)+2 i \omega b_{1}^{c} \alpha_{3}-\alpha_{2}^{c} \Omega_{11}}{\left(1-4 \omega^{2}-2 i \omega a_{1}^{c}\right)\left(2 i \omega-\alpha_{2}^{c}\right)-i \omega \alpha_{2}^{c} \alpha_{1}^{c}} \in \mathbb{C}, \\
B_{32}=\Omega_{32} A_{11} A_{21}, \Omega_{32}=\frac{2 i \omega\left(\Omega_{32}^{\prime} \alpha_{1}^{c}+\alpha_{3} \Omega_{11}\right)}{2 i \omega-\alpha_{2}^{c}} \in \mathbb{C}
\end{array}\right.
\end{gathered}
$$

and

$$
\left\{\begin{array}{c}
\left(1-9 \omega^{2}\right) A_{33}=3 i \omega a_{1}^{c} A_{33}+b_{1}^{c} B_{33}-4 \omega^{2} a_{2} A_{11} A_{22} \\
+2 b_{2} B_{11} B_{22}-i \omega^{3} a_{3} A_{11}^{3}+b_{3} B_{11}^{3} \\
3 i \omega B_{33}=3 i \omega \alpha_{1}^{c} A_{33}+\alpha_{2}^{c} B_{33}+i \omega a_{3}\left(2 B_{11} A_{22}+A_{11} B_{22}\right) .
\end{array}\right.
$$

When equations (15) and (17) are applied

$$
\begin{aligned}
& \Rightarrow A_{33}=\Omega_{33}^{\prime} A_{11}^{3} \text { and } B_{33}=\Omega_{3} A_{11}^{3} ; \text { with } \\
\Omega_{33}^{\prime} & =\frac{i \omega b_{1}^{c} \alpha_{3}\left(2 \Omega_{22}^{\prime} \Omega_{11}+\Omega_{22}\right)}{\left(3 i \omega-\alpha_{2}^{c}\right)\left(1-9 \omega^{2}-3 i \omega a_{1}^{c}\right)-3 i \omega \alpha_{1}^{c} b_{1}^{c}} \\
& +\frac{\left(3 i \omega-\alpha_{2}^{c}\right)\left(2 b_{2} \Omega_{11} \Omega_{22}-i \omega^{3} a_{3}+b_{3} \Omega_{11}^{3}-4 a_{2} \omega^{2} \Omega_{22}^{\prime}\right.}{\left(3 i \omega-\alpha_{2}^{c}\right)\left(1-9 \omega^{2}-3 i \omega a_{1}\right)-3 i \omega \alpha_{1}^{c} b_{1}^{c}} \in \mathbb{C} . \\
\Omega_{33} & =\frac{3 i \omega \alpha_{1}^{c} \Omega_{33}^{\prime}+i \omega \alpha_{3}\left(2 \Omega_{22}^{\prime} \Omega_{11}+\Omega_{22}\right)}{\left(3 i \omega-\alpha_{2}^{c}\right)} \in \mathbb{C} .
\end{aligned}
$$

To compare the $\delta^{3}$ order terms we have

$$
\begin{aligned}
& A_{10}=B_{10}=0, \quad B_{11}=\Omega_{11} A_{11}, \quad B_{20}=\Omega_{20}\left|A_{11}\right|^{2}, \quad A_{20}=\Omega_{20}^{\prime}\left|A_{11}\right|^{2}, \\
& A_{22}=\Omega_{22}^{\prime} A_{11}^{2}, \quad B_{22}=\Omega_{22} A_{11}^{2}, \quad A_{33}=\Omega_{33}^{\prime} A_{11}^{3}, \quad B_{33}=\Omega_{33} A_{11}^{3} \text {. }
\end{aligned}
$$

Also, we know that $B_{31}$ is a function of $A_{11}$ while $\partial_{\tau}$ and $A_{11}$ satisfies (20). In addition

$$
\begin{aligned}
& B_{21}=\Omega_{11} B_{21}, \quad B_{30}=4 \Omega_{30} \operatorname{Re}\left(A_{11} \bar{A}_{21}\right), \quad A_{30}=4 \Omega_{30}^{\prime} \operatorname{Re}\left(A_{11} \bar{A}_{21}\right), \\
& A_{32}=\Omega_{32}^{\prime} A_{11} A_{21}, \quad B_{32}=\Omega_{32} A_{11} A_{21} \text { and } A_{31}=A_{21}=0 .
\end{aligned}
$$

Thus, from equations (19), (25) and (26) $A_{11}$ is a solution of (20) with $B_{31}$ being a function of $A_{11}$ and $\partial_{\tau} A_{11}$ and $A_{10}=B_{10}=A_{21}=B_{21}=A_{30}=B_{30}=$ $A_{31}=A_{32}=B_{32}=0$. Therefore, given a solution of (20), we can evaluate all 
the $A_{k n}$ and $B_{k n}$ solutions for $1 \leq k \leq 3$ and $-k \leq n \leq k$ using the series equations (10) to obtain

$$
\left\{\begin{array}{c}
X_{j}^{A}(t)=\delta A_{11} \lambda+\delta^{2} \Omega_{20}^{\prime}\left|A_{11}\right|^{2}+\delta^{2} \Omega_{22}^{\prime} A_{11}^{2} \lambda^{2}+\delta^{3} \Omega_{33}^{\prime} A_{11}^{3} \lambda^{3}+C_{c}+\mathrm{O}\left(\delta^{3}\right) \\
\psi_{j}^{B}(t)=\delta \Omega_{11} A_{11} \lambda+\delta^{2} \Omega_{20}\left|A_{11}\right|^{2}+\delta^{2} \Omega_{22} A_{11}^{2} \lambda^{2}+\delta^{3} B_{31} \lambda \\
+\delta^{3} \Omega_{33} A_{11}^{3} \lambda^{3}+C_{c}+\mathrm{O}\left(\delta^{3}\right)
\end{array}\right.
$$

\section{Conclusion}

The parameters affecting the existence of periodic solutions generated with rate and state friction in spring-block model were stated. Derivation of the modulation equation for the model with non-linear rate-and state- friction law that allows the amplitude of the wave to vary slowly with time and space was presented. Conditions under which the modulation wave equation produced many small solutions near the instability threshold for finitely large enough time scales were established.

\section{References}

[1] S.T. Tse, J.R. Rice, Crustal earthquake instability in relation to the depth variation of frictional slip properties, J. Geo phys. Res., 91 (1986), 94529472.

[2] C.R. Burridge, L. Knopoff, Model and theoretical seismicity, Bull. Seism. Soc. Am., 57 (1967), 341-371.

[3] K. Annan, Existence and stability analysis of spring-block model solutions with rate and state friction, Applied Mathematical Sciences, 7, No. 36 (2013), 1785-1795.

[4] J.M. Carlson, J.S. Langer, B.E. Shaw, Dynamics of earthquake faults, Reviews of Modern Physics, 66 (1994), 657.

[5] U. Galvanetto, Sliding bifurcations in the dynamics of mechanical systems with dry friction - remarks for engineers and applied scientists, J. Sound Vibr., 276 (2004), 121-139. 
[6] K. Annan, Propagation of local and global smoothed periodic waves in a spring-block model, IJPAM, 81, No. 3 (2012), 439-461.

[7] J.R. Rice, A.L. Ruina, Stability of steady frictional slipping, J. Appl. Mech., 50 (1983), 343-349.

[8] J.H. Dieterich, Modeling of rock friction: Experimental results and constitutive equations, J. Geophys. (1979); Res., 84(B5), 2161-2168, doi: 10.1029/JB084iB05p02161.

[9] A. Ruina, Slip instability and state variable friction laws, J. Geophys. (1983); Res., 88 (B12), No. 10, doi: 10.1029/JB088iB12p10359.

[10] S.T. Tse, J.R. Rice, Crustal earthquake instability in relation to the depth variation of frictional slip properties, J. Geo phys. Res., 91 (1986), 94529472 .

[11] J.R. Rice, Spatiotemporal complexity of slip on a f ault, J. Geophys. Res., 98 (1993), 9885-9907.

[12] C. Marone, Laboratory-derived friction laws and their application to seismic faulting, Annu. Rev. Earth Planet., 26 (1998), 643-696.

[13] J.H. Dieterich, A constitutive law for rate of earthquake production and its application to earthquake clustering, J. Geophys. Res., 99 (1994), 26012618.

[14] J.H. Dieterich, V. Cayol, P. Okubo, The use of earthquake rate changes as a stress meter at Kilauea volcano, Nature, 408 (2000), 457-460.

[15] S. Toda, R.S. Stein, T. Sahiya, Evidence from the AD 2000 Izu islands earthquake swarm that stressing rate governs seismicity, Nature, 419 (2002), 58-61.

[16] R.S. Stein, Earthquake conversations, Sci. Am., 288 (2003), 72-79.

[17] T. Baumberger, C. Caroli, Solid friction from stick-slip down to pinning and aging, Adv. in Phys., 55 (2006), 279-348.

[18] T. Putelat, J.H.P. Dawes, J.R. Willis, Regimes of frictional sliding of a spring-block system, J. Mech. Phys. Solids, 58 (2010), 27-53.

[19] T. Putelat, J.H.P. Dawes, J.R. Willis, On the microphysical foundations of rate-and-state friction, J. Mech. Phys. Solids, 59 (2011), 1062-1075. 
[20] T. Putelat, J.R. Willis, J.H.P. Dawes, On the seismic cycle seen as a relaxation oscillation, Phil. Mag., 88 (2008), 3219-3243.

[21] E. Rabinowicz, The intrinsic variables affecting the stick-slip process, Proc. Phys. Soc., London, 71 (1957), 668-675.

[22] A. Ohmura, H. Kawamura, Rate-and-state-dependent friction law and statistical properties of earthquakes, Europhysics Letters, 77, No. 6 (2007).

[23] A. Mielke, The Ginzburg-Landau Equation in its Role as a Modulation Equation, Handbook of dynamical systems Vol. 2, Elsevier Science. 